Impulsivity and Behaviour Problems in Dogs: A Reinforcement Sensitivity Theory Perspective.

\title{
Reinforcement Sensitivity Theory of Personality Questionnaire - Dog (RSTPQ-D)
}

This measure is designed to assess the Reinforcement Sensitivity Personality theory traits in dogs. It is for use by owners or researchers to code the behaviour of dogs they know well.

If using this tool, please cite the measure as the below (or a more recent version):

Piotti, P., Satchell, L., \& Lockhart, T. (2018, February 1). Impulsivity and Behaviour Problems in Dogs: A Reinforcement Sensitivity Theory Perspective. Retrieved from psyarxiv.com/sutdv

Please note this version of the questionnaire is pre-validation.

\section{Background in brief.}

It is recommended that individuals interested in using the RSTPQ-D should read Piotti et al (2018) first.

"[Reinforcement sensitivity theory] is a neuropsychological account of the neural and cognitive processes underlying the major dimensions of personality (Corr, 2008). The theory describes three neurologically defined systems which influence the organism's behaviours; the Behavioural Approach System (BAS, activated by signals of reward), the Behavioural Inhibition System (BIS, related to the monitoring and resolution of conflict between compelling goals) and the Fight-Flight-Freeze System (FFFS, activated by aversive stimuli)." (Piotti et al., 2018, p.3-4)

Dispositional BAS is related to reward seeking and impulsive behaviours. This has been shown in correlations between the RSTPQ-D's BAS measures and other established measures of dog impulsivity (see Piotti et al., 2018).

Trait BIS is related to increased passive avoidance behaviours (such as shaking, avoiding others) and decreased destructive behaviours (such as damaging objects, digging) in dogs (see Piotti et al., 2018).

A dog who had stronger FFFS traits was less prone to active avoidance behaviours (such as snapping, biting, growling) and more prone to passive avoidance behaviours (see Piotti et al., 2018).

\section{Norms (see table 1).}

"Responses from owners of 730 adults dogs (age range: 1 - 16 years, median $=5$ years, $S D=3.36, \mathrm{M}: \mathrm{F}=1$, neutered $:$ intact $=6: 1$ )" were used to establish norms. (Piotti et al., 2018, pages 8-7).

"The RSTPQ-D retained an acceptable fit for its factor structure (21 items into three domains of FFFS, BIS and BAS) when applied to the owner's ratings of dogs (CFA: $X^{2}(d f=186)=1001.94, p<0.001, C F I=0.88$, RMSEA $=$ 0.08)." (Piotti et al., 2018, page 9). 
Impulsivity and Behaviour Problems in Dogs: A Reinforcement Sensitivity Theory Perspective.

Table 1. The descriptive statistics of the Reinforcement Sensitivity Theory Personality Questionnaire - Dog traits

\begin{tabular}{lllll}
\hline Variable & Mean & SD & Skewness & Kurtosis \\
\hline Behavioural Approach System & 3.81 & 0.81 & -0.58 & -0.08 \\
Behavioural Inhibition System & 2.92 & 1.07 & -0.04 & -0.89 \\
Fight/Flight/Freeze System & 2.46 & 0.84 & 0.29 & -0.23 \\
\hline
\end{tabular}




\begin{tabular}{|c|c|c|c|c|c|c|}
\hline & $\begin{array}{l}\text { Reinforcement Sensitivity Theory of Personality } \\
\text { Questionnaire - Dog }\end{array}$ & 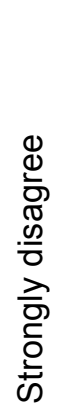 & 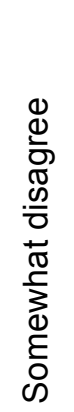 & 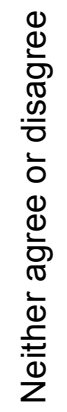 & 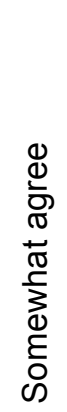 & 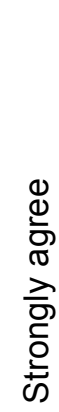 \\
\hline 1. & $\begin{array}{l}\text { My dog would be frozen to the spot if there was a } \\
\text { dangerous animal in the house with him/her. }\end{array}$ & 1 & 2 & 3 & 4 & 5 \\
\hline 2. & $\begin{array}{l}\text { My dog would be frozen to the spot if he/she saw a large } \\
\text { shadow when swimming. }\end{array}$ & 1 & 2 & 3 & 4 & 5 \\
\hline 3. & $\begin{array}{l}\text { My dog would run away if he/she saw a dangerous } \\
\text { animal. }\end{array}$ & 1 & 2 & 3 & 4 & 5 \\
\hline 4. & $\begin{array}{l}\text { My dog would freeze if he/she thought a something was } \\
\text { going to attack him/her. }\end{array}$ & 1 & 2 & 3 & 4 & 5 \\
\hline 5. & $\begin{array}{l}\text { My dog would freeze if he/she heard scary noises at } \\
\text { night. }\end{array}$ & 1 & 2 & 3 & 4 & 5 \\
\hline 6. & $\begin{array}{l}\text { My dog would run away from an animal if it was making } \\
\text { her/him feel scared. }\end{array}$ & 1 & 2 & 3 & 4 & 5 \\
\hline 7. & $\begin{array}{l}\text { My dog would run upstairs if there was something scary } \\
\text { downstairs. }\end{array}$ & 1 & 2 & 3 & 4 & 5 \\
\hline 8. & $\begin{array}{l}\text { My dog is careful when doing something that might hurt } \\
\text { him/her. }\end{array}$ & 1 & 2 & 3 & 4 & 5 \\
\hline 9. & My dog would be careful when playing. & 1 & 2 & 3 & 4 & 5 \\
\hline 10. & $\begin{array}{l}\text { My dog would stop what he/she was doing if he/she } \\
\text { thought there was physical danger or he/she might hurt } \\
\text { him/herself. }\end{array}$ & 1 & 2 & 3 & 4 & 5 \\
\hline 11. & $\begin{array}{l}\text { My dog would stop what he/she was doing if he/she } \\
\text { thought it was too risky to keep going. }\end{array}$ & 1 & 2 & 3 & 4 & 5 \\
\hline 12. & My dog worries about getting hurt. & 1 & 2 & 3 & 4 & 5 \\
\hline 13. & $\begin{array}{l}\text { My dog would stop and think before going down a steep } \\
\text { slope or sharp drop (where they would not be able to } \\
\text { stop easily). }\end{array}$ & 1 & 2 & 3 & 4 & 5 \\
\hline 14. & $\begin{array}{l}\text { My dog appears to stop and think carefully before trying } \\
\text { out for something. }\end{array}$ & 1 & 2 & 3 & 4 & 5 \\
\hline 15. & $\begin{array}{l}\text { My dog spends a lot of time trying to get better at things } \\
\text { he/she likes doing (such as fetch/agility). }\end{array}$ & 1 & 2 & 3 & 4 & 5 \\
\hline 16. & $\begin{array}{l}\text { My dog works hard to do well at the things they like } \\
\text { doing (like playing 'find it' or 'fetch'). }\end{array}$ & 1 & 2 & 3 & 4 & 5 \\
\hline 17. & My dog likes to practice something he/she likes doing. & 1 & 2 & 3 & 4 & 5 \\
\hline 18. & $\begin{array}{l}\text { My dog puts in lots of effort to achieve a goal (or get } \\
\text { what he/she wants). }\end{array}$ & 1 & 2 & 3 & 4 & 5 \\
\hline 19. & $\begin{array}{l}\text { My dog wants to keep on improving (getting better) at } \\
\text { his/her favourite things. }\end{array}$ & 1 & 2 & 3 & 4 & 5 \\
\hline 20. & My dog is interested in exploring places. & 1 & 2 & 3 & 4 & 5 \\
\hline 21. & My dog likes to do new and exciting things. & 1 & 2 & 3 & 4 & 5 \\
\hline
\end{tabular}


Impulsivity and Behaviour Problems in Dogs: A Reinforcement Sensitivity Theory Perspective.

\section{Factors and analysis.}

Average scores to trait domains are retained for analysis.

There are no reversed scored items.

Fight/Flight/Freeze System trait - Average response to questions 1-7

Behavioural Inhibition System trait- Average response to questions 8-14

Behavioural Approach System trait - Average response to questions 15-21 